\title{
Characterisation of the Effect of Corrosion on the Residual Stresses in Girth Weld Pipe Using Ultrasonic Calibrated with Strain-relieving Measurement Techniques
}

\author{
R. Romac ${ }^{1 a^{*}}$, D. Cave $^{1 \mathrm{~b}}$, X. Ficquet ${ }^{1 \mathrm{c}}$ \\ ${ }^{1}$ VEQTER Ltd, 8 Unicorn Business Park, Whitby Road, Bristol BS4 4EX, UK \\ aremi.romac@veqter.co.uk, bdouglas.cave@veqter.co.uk, cxavier.ficquet@veqter.co.uk
}

\begin{abstract}
Keywords: Residual Stress, Girth Weld, Ultrasonic Measurement, Contour, iDHD, Corrosion
\end{abstract}

\begin{abstract}
The structural integrity of an oil and gas pipeline is increasingly factored into the design of new installations to ensure that operating risks are maintained low. In addition, the life extension of existing assets beyond their original anticipated design life, as a result of the current oil price environment and the need to optimize field development expenditure, is an ongoing challenge. Operators would like to extend pipeline service life, while many of the technologies required for the validation of their ongoing condition are not yet mature enough to provide confidence that this is a viable strategy. One of the issues considered as a key threat to pipeline integrity is corrosion. Therefore understanding the distribution and redistribution of residual stresses within a pipeline affected by corrosion can be of great benefit. A way to monitor in-situ the pipeline residual stress is to use the ultrasonic (UT) technique. The paper aimed to assess and calibrate the US technique on a pipeline mock-up in the presence of a typical local corrosion damage mechanism. Contour, iDHD, ICHD, XRD and ultrasonic measurements were carried out before machining a flaw to produce an accurate FE model of the pipe. The residual stress was then measured during the manufacture of the flaw and was compared with the FE prediction. Ultrasonic measurements were then carried out on the outer surface of the pipe and show a significant increase in the residual stress. The Ultrasonic technique can therefore, be used to monitor the changes in the residual stress which may be caused by corrosion.
\end{abstract}

\section{Introduction}

Across the globe, 5 metric tons of steel are degenerated every second with the offshore industry. The main fault on pipelines in the North Sea and the Gulf of Mexico is caused by internal corrosion. About $67 \%$ of the global pipeline are now older than 20 year which is the minimum design life [1]. Guideline provides guidance to estimate the life of a component in the presence of a flaw, analytically or with finite element analysis [2]. The finite element analysis can be less conservative than the analytical solution and, therefore, increase the in-service time of the pipeline. In order to create a representative FE model of the pipeline, an accurate description of the weld residual stress is important. In this study, the Contour, the Incremental Deep Hole Drilling (DHD), the Incremental Center-Hole Drilling (ICHD) and XRD residual stress measurement techniques were employed and compared on an as-welded section of pipeline.

In order to evaluate the life of a pipeline, the corrosion growth needs to be known. Ultrasonic technique is already used to measure the wall thickness of the pipes. This study, however, looks at the measure of the residual stress using ultrasound in an as-welded zone and in a zone with thinner wall thickness. To obtain this results the ultrasonic system needs to be first calibrated. The calibration can be done using a tensile test or using absolute residual stress values. In this study, the ultrasonic method was calibrated using strain relieving technique. 


\section{The Ultrasonic technique}

The measurement of residual stress with Ultrasonic is mainly done via standard crack detection ultrasonic probes. The primary difference is about the way to use the probes. For each measurement, the pulse is sent to a transmitter probe via a Pulser-Receiver device. The voltage signal is sent to a Piezo-electric probe which vibrates and produces an ultrasonic wave sent into the Acrylic wedge. This wave travels through the acrylic wedge at a lower speed than the metallic material tested. As it comes out of the probes, the direction of propagation of the wave is parallel to the probe. Once it reaches the specimen, it starts to travel at an angle equal to the sinus of the wedge angle divided by the speed of the longitudinal ultrasound in the acrylic material, then multiplied by the speed of the longitudinal ultrasound in the specimen, as per the formula below:

$$
\frac{\sin \theta_{1}}{V_{L w}}=\frac{\sin \theta_{2}}{V_{L s}}
$$

Where $\theta_{1}$ is the angle from the ultrasonic emitting probe to the normal to the specimen surface, $\theta_{2}$ is the angle from the normal of the specimen surface to the refracted wave, and $V_{L w}$ and $V_{L s}$ are the material longitudinal ultrasonic velocity respectively for the wedge and the specimen.

If the angle of the probe is appropriately set-up on the wedge - which depends on the material tested - then the wave travels below the surface of the specimen at an angle parallel to the surface of the specimen. The wave is then picked up by a first receiver, distant from around $18.2 \mathrm{~mm}$ and the second one around $34 \mathrm{~mm}$ from the emitter probe. Both probes receive the longitudinal critically refracted (LCR) wave but at different times. The arrival time for each probe is measured and the difference of Time-of-Flight between the two probes are then calculated. Fig. 1a shows the cross section of the acrylic wedge.
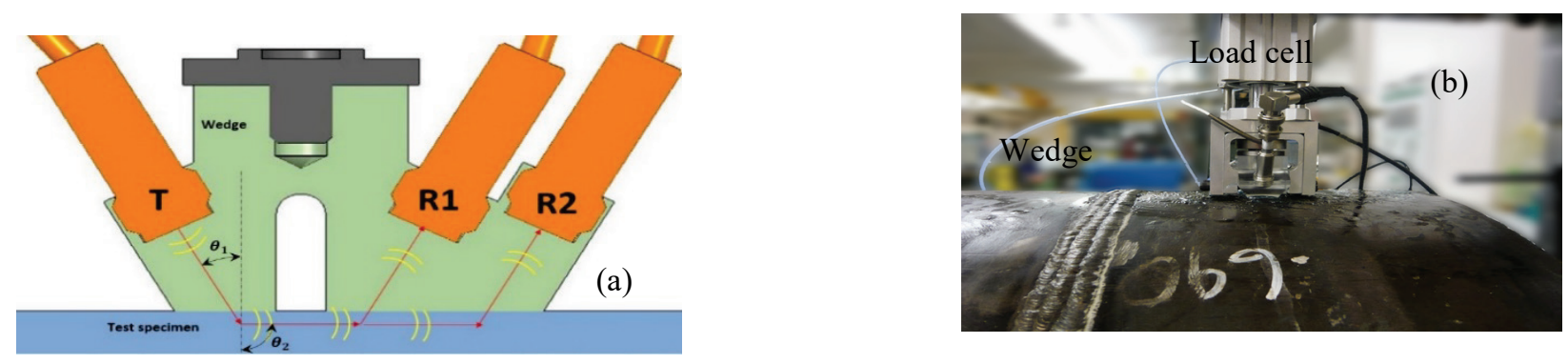

Fig. 1: (a) Diagram showing the Lcr technique principle, (b) Setup of the ultrasonic measurement

In order to calculate the residual stress, the following formula is used:

$$
\Delta \sigma=\frac{E(t-t 0)}{\text { L11.t0 }}
$$

L11 is called the acoustoelastic coefficient and translate of the slope of the difference of residual stress with a changing Time-of-Flight [3]. It is inherent to the material. E is the Young's modulus, $t$ is the recorded Time-of-Flight (TOF) for the current measurement and t0 is the recorded Time-of-Flight at the initial state or stress-free state. For the Young's Modulus, a fixed value of 203GPa was used.

In order to determine the Time-of-Flight, several analyses are being used for the calculation. The common way of determining the Time-of-Flight between two ultrasonic signals is by picking up a specific event in the signal that could be comparable with different signals. The point must be an event that is equally happening for each echo and is only alterable because of the residual stress [4]. 


\begin{abstract}
Absolute Measurement technique description
Absolute residual stress measurements were carried out on the pipe in order to determine the full residual stress field. The iDHD, Contour, ICHD and XRD techniques were used.

The iDHD technique can provide bi-axial residual stress through the thickness of the joint [5]. This technique can measure steep gradient through the thickness, however the results are only provided along the measurement line and documented in [6].

The Contour residual stress measurement technique is a destructive mechanical strain relief technique. The technique is based on a variation of Bueckner's elastic principle of superposition [7] and consists of three main stages. The technique provides a full uniaxial map of the residual stress perpendicular to a cut plane through the specimen. Therefore providing the location of the maximum and minimum residual stress peaks. The results are also documented in [6].

The XRD technique was used to provide non-destructive "surface" measurements, giving a single measurement data point which represents the average residual stresses acting within the first $10 \mu \mathrm{m}$ of the component surface.

The ICHD technique [6] provided residual stress profiles from $0.05 \mathrm{~mm}-1.00 \mathrm{~mm}$ depth at each location. As mentioned previously, the ICHD measurements were carried out according to the ASTM standard assuming an isotropic linearly elastic material and with a plane stress condition.
\end{abstract}

\title{
Component description and measurement locations
}

The pipe specimen was made from ferritic steel API 5L X52. As illustrated in Fig. 3b, the specimen had a length of about $410 \mathrm{~mm}$ and an outer diameter of $762 \mathrm{~mm}$. It contained a seam weld along the length of the pipe and a circumferential girth weld at mid-length of the pipe. A cut-out had been machined on the inner diameter at mid-length and has an axial length of about $150 \mathrm{~mm}$, reducing the thickness of the pipe from $25 \mathrm{~mm}$ to about $19 \mathrm{~mm}$ in the weld area. It was assumed that both the weld and parent metals had the same material properties with a Young's modulus of 203GPa, a Poisson's ratio of 0.3 and a yield strength of $450 \mathrm{MPa}$.

A total of 75 XRD measurements were carried in the ID and OD across the weld at loc 1 and Loc2. In this paper only the measurements carried on the weld centreline (WCL) and the heat affect zone (HAZ) at $20 \mathrm{~mm}$ from the WCL in the OD and at $10 \mathrm{~mm}$ from the weld root in the ID are presented. Three ICHDs were performed on the OD at LOC2, on the WCL, 20mm away from the WCL and $38 \mathrm{~mm}$ away from the WCL for ICHDs 1, 2 and 3 respectively. The three other ICHDs were performed at LOC2 but on the inner diameter, on the WCL, $10 \mathrm{~mm}$ and $30 \mathrm{~mm}$ away from the WCL for ICHDs 4, 5 and 6 respectively.

Two iDHDs were performed at two specific locations: at the WCL and in the HAZ in order to determine the through thickness residual stresses induced by the welding process. Since ICHDs have been performed at LOC2, iDHD A, was performed at LOC1 so it could be drilled at a location where the weld hadn't been polished. It was performed through the WCL and drilled from the outer diameter of the pipe, with a hole diameter of $1.5 \mathrm{~mm}$ and extracting a $5 \mathrm{~mm}$ core. iDHD B was performed at LOC $3,90^{\circ}$ from the seam weld and at $10 \mathrm{~mm}$ axially from the WCL.

The Contour measurement location was an axial-radial plane through the pipe, opposite the initial pipe splitting saw cut location. The cut was arranged such that the contour surfaces would permit the hoop residual stresses to be measured.

\section{Destructive residual stress measurements results and discussions}

The results of the absolute measurements for the hoop direction can be seen in Fig. 2. Fig. 2a shows the through thickness residual stress at the WCL location. At the near OD surface the XRD results show a low compression value which rapidly increase to high tension as shown by the ICHD results. The ICHD results are clearly overestimating the hoop stress. The ICHD tend to overestimate the residual stress when its value are near the yield strength. The iDHD and contour show high tensile stress throughout the thickness of the weld. The iDHD gave value near yield stress while the contour 
result was lower. The last ICHD measurement carried out at the ID agreed very well with the iDHD measurement.

Fig. $2 \mathrm{~b}$ shows the through thickness residual stress at the HAZ location 10mm from the WCL. Again the XRD measurements show some low compression value near the OD and ID surfaces. Which raise rapidly to high tension value as shown by both ICHD measurement. At this location both Contour and iDHD provide the same results with higher tension value about $400 \mathrm{MPa}$ at the OD decrease slowly toward the ID to values around $250 \mathrm{MPa}$.
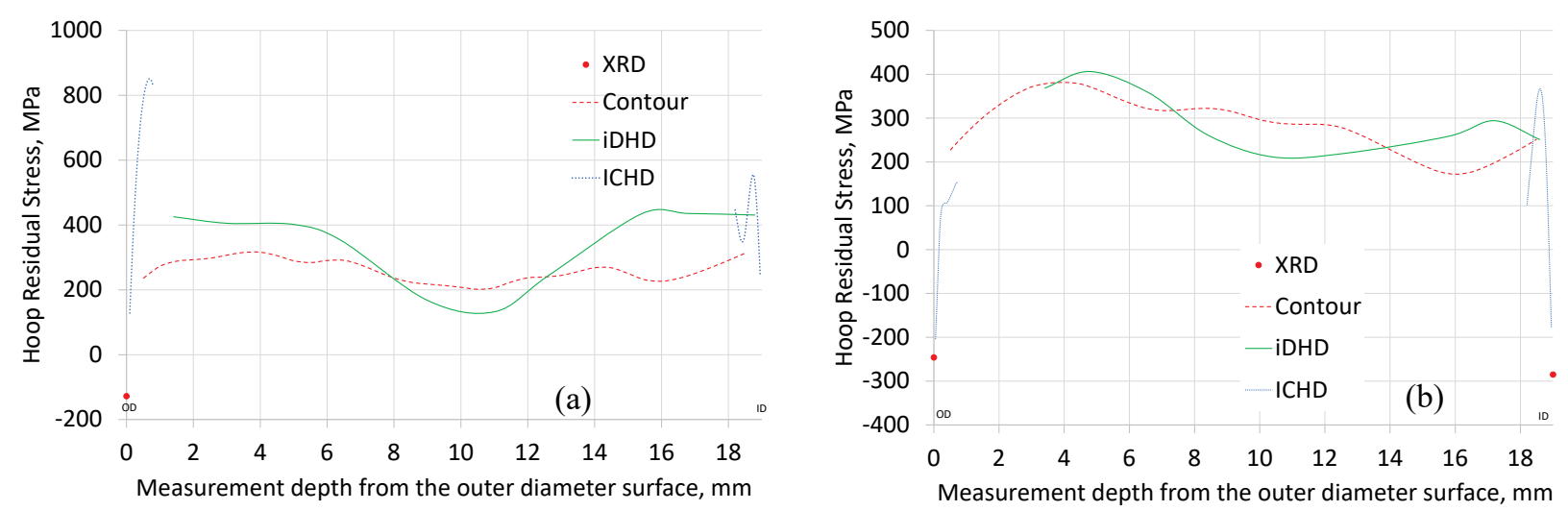

Fig. 3 a shows the hoop residual stress from the contour measurement in a 2D map. High tensile stress is found at the girth weld location with a higher tensile value near the HAZ than in the $W C L$. The iDHD measurement however, has shown that at this location the tensile stress is in the same order of magnitude. Compression residual stress balancing the tensile stress can be seen from $25 \mathrm{~mm}$ away from the WCL.

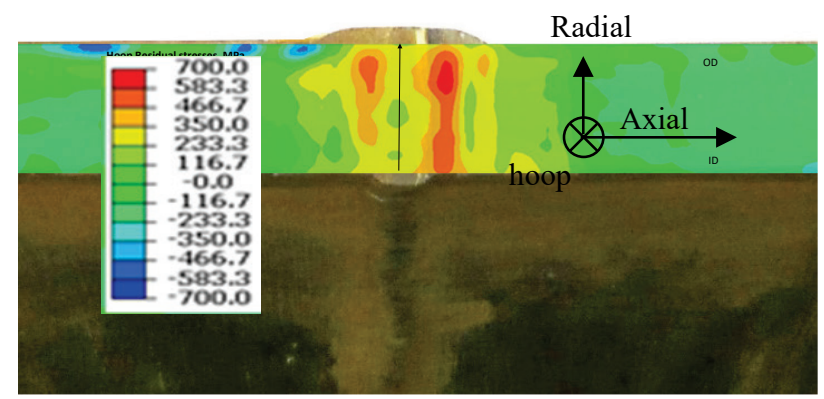

(a)

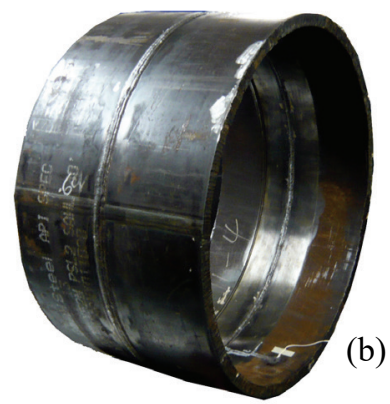

Fig. 3: (a) 2D map of the contour measurement, (b) Photograph of the specimen

\section{Ultrasonic residual stress measurement results and discussions}

There are several sources of uncertainty when measuring the residual stress using ultrasonic waves. One of the difficulty is the accuracy of the time-of-flight measurement. Indeed, although it was found that longitudinal waves are the most sensitive to residual stress [3], the resolution needed to detect a change in the acoustic speed is generally high. A material with an acoustoelastic coefficient of 1 would mean that $100 \mathrm{MPa}$ would represent a change of around $7 \mathrm{~ns}$. As a consequence, it is necessary that the signal acquisition device is accurate enough. The pulse is sent via an ultrasonic pulser at $15 \mathrm{~Hz}$ into the transmitter probe. It is also sent towards a fast sampling oscilloscope to set-up the trigger reference point. The oscilloscope then displays the longitudinal critically refracted echoes picked-up by the two receivers at a resolution of $0.4 \mathrm{~ns}$. The uncertainty due to the sampling equipment was therefore found to be $\pm 1.5 \mathrm{MPa}$ in the hoop direction which is very low. 
Other sources of error when measuring can occur such as the temperature, the texture and the couplant thickness. It was seen [4] that a change of temperature can significantly affect the acoustic speed. Therefore, the temperature was monitored in lab condition for each test using a data logger and several thermocouples and was found to be constant around $21^{\circ} \mathrm{C}$.

When using the contact method, ultrasonic gel needs to be applied at any interfaces between the wedge, the transducers and the specimen in order to allow the wave to be transmitted into the material to reach the receivers. Uncertainties can however occur if the thickness of this gel is not properly controlled. The slightest change in couplant thickness can induce hundreds of $\mathrm{MPa}$ differences. In order to minimize errors associated with couplant thickness, constant and consistent pressure needs to me maintained on the wedge. As it can be seen in Fig. 1b this was controlled by using a pneumatic ram and a load cell to monitor the force to be $179.9 \pm 7.3 \mathrm{~N}$ in the hoop direction. The probes used were screw-in Time-Of-Flight diffraction probes and therefore the gel thickness and contact between the probes and the acrylic wedge remained constant throughout the test. Finally, the two regions examined were compared against each other and so parent vs parent, HAZ vs HAZ and weld vs weld, therefore the textures should be comparable.

\section{Calibration of the ultrasonic measurement}

In order to determine the acoustoelastic constant $\mathrm{L}_{11}$ and $\mathrm{t}_{0}$ a tensile test need to be carried out on a dog bone type stress-free sample extracted from the studied sample. The objective would be to measure the acoustic velocities for different applied load. A uni-axial strain gauges would be fitted on the tensile specimen to determine the stress due to the load. Hence, one could accurately determine the relation between the Time-of-flight measured and the stress in the material. However, this test can be avoided if the $\mathrm{L}_{11}$ constant is calibrated with absolute residual stress measurement which is the aim of this study. Therefore Incremental Centre Hole Drilling measurements averaged over their depths have been used to calibrate the acoustic velocities to the absolute residual stress magnitudes. Fig. 4a shows the calibrated ultrasonic measurements compared with the iDHD, ICHD and contour measurements. It can be seen that there is very good agreement with the DHD and Ultrasonic results. The acoustoelastic constant for this material was found to be 4.1 in the hoop direction, which is in the range of other materials tested. The calibration process also improved the determination of the stress free wave velocity in the hoop direction in this material and so providing a vertical datum, or stress free reference to adjust the overall levels by.

Once the ultrasonic calibration has been done, there is high interest in to measuring a different state of stress, i.e. weld corrosion, in order to characterise any residual stress changes which is the subject of the next section.

\section{Corrosion simulation}

Offshore pipelines are subject to internal corrosion reducing the structural integrity of the pipe. An, example corrosion mechanism to be considered is Preferential Weld Corrosion. Typically, the deepest PWC zones cover about 30\% of the circumference of the pipe.

A study was carried out to evaluate the change in residual stress as a groove was incrementally machined out to about $5-6 \%$ of the pipe circumference. In order to measure the change in the residual stress field a technique similar to the DHD was used. A $1.5 \mathrm{~mm}$ diameter hole was created and measured as the groove was machined as seen in Fig. 4b. The results of this work are not presented in this paper.

After the final groove increment at about 95\% deep. Ultrasonic were carried out across the weld near the groove location (LOC4) and compared with the previous measurement without the groove, shows in Fig. 4b. The ultrasonic measurement used the same calibration found before the groove. The results show a much higher tensile peak of about $1500 \mathrm{MPa}$ than the as welded pipe location. The ultrasonic measurement after the groove seems to overestimate the residual stress. However, a clear difference in residual stress can be observed using a non-destructive technique of a pipe with a 
groove i.e. with internal corrosion than an intact pipe. Further work could investigate the magnitude of the peak with different groove depth and geometries.

\section{Conclusion}

A residual stress study was carried out on a $762 \mathrm{~mm}$ diameter and $19 \mathrm{~mm}$ thick pipe representative of an offshore pipeline. Comprehensive measurements were carried out to determine the full residual stress field using the Contour, the iDHD, the ICHD and XRD. It was found that all four techniques were in good agreement. Using the ICHD technique, the ultrasonic method was calibrated to also provide absolute residual stress across the weld on the OD. A very good agreement was found between the ultrasonic measurement and the other destructive techniques. Offshore pipelines are subject to corrosion. A groove was machined internally to simulate some corrosion. The calibrated ultrasonic machine was then used on the groove location to measure the change of residual stress. The residual stress at the groove location was measured and found to be much higher than the measured stress at an intact location. The magnitude of the measured stress at the groove location was overestimated, however, the non-destructive technique clearly identifies a rise in the residual stress. Further investigation should be performed to characterise the overestimation.
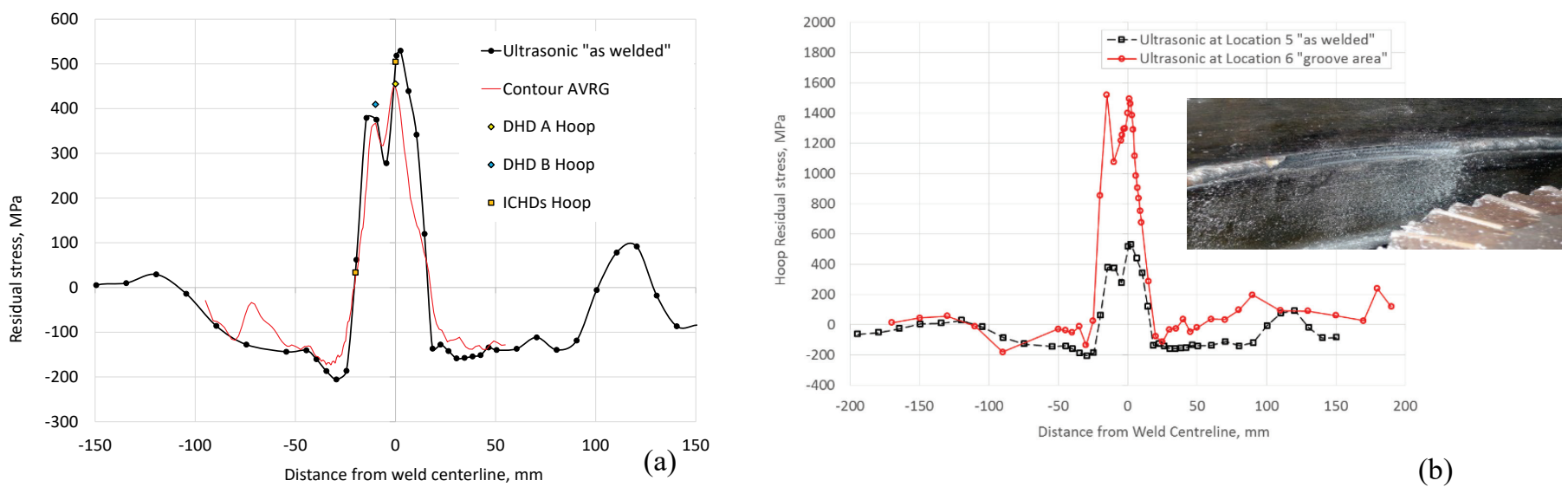

(b)

Fig. 4: Comparison of the ultrasonic measurement across the weld, (a) without grove compared with contour and $i D H D$, (b) with and without the presence of a groove

\section{Acknowledgements}

The authors would like to thank Dale McIntyre and Herman Rincon from ConocoPhillips (U.S.A) for providing the mock-up and supporting this research.

\section{References}

[1] DNV, recommended practice det norske veritas dnv-rp-f116, "Integrity management of submarine pipeline systems", (2009)

[2] BS7910, "Guide on methods for assessing the acceptability of flaws in metallic structures", British Standard Institution. (2005)

[3] A.A. Santos, D.E. Bray, "Ultrasonic Stress Measurements using PC based and commercial flaw detector", Review of Scientific Instruments, (2000). http://dx.doi.org/10.1063/1.1287339

[4] Y. Javadi, H. S. Pirzaman, M. H. Raeisi, M. A. Najafadabi, Ultrasonic inspection of a welded stainless steel pipe to evaluate residual stresses through thickness, (2013)

[5] Mahmoudi, A.H. et al., "A New Procedure to Measure near Yield Residual Stresses Using the Deep Hole Drilling Technique”, Journal of Experimental Mechanics, , Vol. 49, No. 4, (2009)

[6] Serasli, $\mathrm{K}$ et al., "Measurements of residual stresses using mechanical strain relaxation methods and mapping in a thin-walled girth welded pipe mock-up, Pressure Vessels \& Piping conference 2016

[7] Hosseinzadeh, F. et al. "Towards good practice guidelines for the contour method of residual stress measurement", Journal of Engineering (2014) 